\title{
WASFM: A Web Application System Functional Model
}

\author{
C.C. Zhu, J.P. Xiu, Z.Q. Yang \\ Beijing University of Posts and Telecommunications \\ Beijing 100876, China
}

\begin{abstract}
Web application system is a kind of system organization form which is of large scale and complex. Accurate requirements modeling of this kind of system has very important effect on system success. A web functional modeling method is raised in this paper called WASFM. The paper presents the formal description of WASFM and proves the efficiency of this model through a web system modeling example.
\end{abstract}

Keywords-web application functional model; formal language; function type

\section{INTRODUCTION}

The web application system (WAS) is a kind of network software which does not need to install client program and can be accessed by browser, so WAS is also called $\mathrm{B} / \mathrm{S}$ system. WAS is an advanced system architecture owning some obvious advantages like the economic efficiency in system development and the flexibility in system extension. That's why it has become the trend of e-commerce, egovernment and other life services. The most famous web systems include 'Baidu', 'Google', 'taobao', 'facebook' and so on.

Web application systems are always complicated with a large number of functions, so system requirement analysis is the emphasis and the difficulty in system construction. Clear and detailed definitions of system requirements are very important for system design, development and testing work. Functional model needs to define the system function type, page layout, field definitions, handle constraint and business logic, etc. This paper is focused on the research of WAS functional modeling methods and proposed a formal model named WASFM.

\section{RESEARCH STATUS}

The common function modeling methods include UML modeling method, IDEF0 modeling method and GTST method, etc.

UML (Unified Modeling Language) is the standard modeling language widely used in the field of object-oriented technology, it allows the visual representation of a system's specification at various levels of design, and it is used to construct and document the artifacts of an object-oriented software system [1]. There are many modeling tools in UML like Use Case Diagram, Class Diagram, State Diagram, and Sequence Diagram, etc. The Use Case Model is a kind of high level functional modeling tool to display the relationships between actors and use cases [2]. But when we want to define a function as adding a new user, we want to describe the fields like: the name of the field, the form controller it uses, the initial values and the constraints. But the Use Case Description has no formal specifications; it can't describe the functional requirements in details.

IDEF0 is based on structured analysis method, and adopts the strict top-down way to construct the model step by step. IDEF0 uses abbreviations, namely ICOM (I for Input, C for Control, O for Output and $\mathrm{M}$ for Mechanism) to refer to the elements of a function [3]. Unlike other notations that only concern information, IDEF0 covers a number of aspects including information, roles and means of actions [4]. But it cannot describe the details of a function such as the entity the function corresponds.

The GTST (Goal-tree Success-Tree) method is based on the data flowing GTST method, the system is divided into small goals, the way to the deepest level is called goal tree, and the description of the way to realize the base goal is called the success tree [5]. It contains goals, conditions and attributes in its description so people can understand the system well. But it is suitable for the simple system. When it comes to the complicated system, the model is too large; it is not easy to understand the functions through the model.

In this paper we use formal language to define the functional model for WAS which contains as many function definitions as possible. This model will guide the requirement analysis of WAS, lay the fundamental for the design, shorten the cycle of development, improve the testing efficiency and finally achieve the aim of ensuring the quality of the system.

\section{MODEL DEFINITIONS}

Based on study of the existed methods and analysis of a lot of WAS structures, this paper divides the typical functions into three types. They are:

(1)The catalog function: it corresponds to the navigation links of WAS to arrange the system contents to different modules

(2)The page function: it is a functional link corresponds to a specific web page in the WAS.

(3)The button function: it is a functional button corresponds to a specific operation. The common button functions include: add, delete, edit, check, import and export, etc. 
WAS is a collection of the three types of functions. As shown in Figure 1, WAS should include many catalog functions and catalog function should include sub catalog functions or page functions. Page function should contain many operation area in which located many button functions. Button function should start some system process, for example insert a new user, delete an order, search some goods or pay some account.

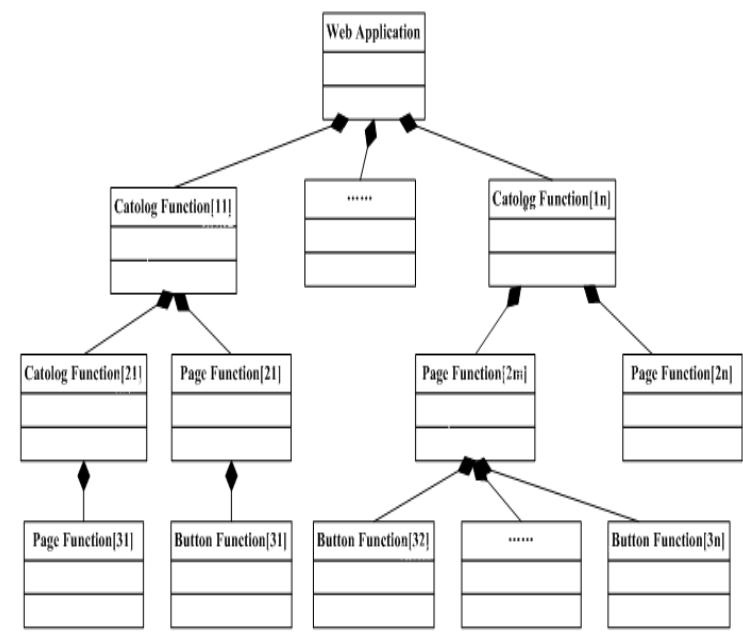

FIGURE I. WEB APPLICATION FUNCTION STRUCTURE DIAGRAM.

In order to describe the function model for WAS accurately, this paper proposes a formal WAS functional model method called WASFM. The specific contents are as below.

Definition 1: WAS: Web Application System is an $\Sigma$ (FR, NFR), where:

1) FR is a set of functional requirements, and $F R \neq \varnothing$

2) NFR is a set of non-functional requirements.

Note: The focus of this paper is FR model method, so the NFR model methods won't be considered.

Definition 2: FR: Functional Requirements is an $\Sigma(\mathrm{CF}$, PF, BF), where:

1) $\mathrm{CF}$ is a set of catalog functions

2) $\mathrm{PF}$ is a set of page functions.

3) $\mathrm{BF}$ is a set of button functions.

Definition 3: CF: Catalog Function is an $\Sigma$ (Name, Level, PAF), where:

1) Name is the function name

2) Level is the level of the function.

3) PAF is the parent function. If the function is the top level, PAF value is 0 .

Definition 4: PF: Page Function is an $\Sigma$ (Name, URL, PAF, PC), where:

1) Name is the function name
2) URL is the page access address

3) PAF is the parent function. If the PF is the main page, the PAF value is 0 .

4) $\mathrm{PC}$ is the Page Contents which describes the contents that the web page should include. $\mathrm{PC}$ is a set of $\mathrm{AD}$.

Definition 5: AD: Area Description is an $\Sigma$ (ID, Position, AT, AC), where:

1) ID is the area unique identifier in the page

2) Position is the area location in the page. Position is an $\Sigma$ (Top, Left, Bottom, and Right), where:

a) Top is the ordinate of the upper left corner

b) Left is the abscissa of the upper left corner

c) Bottom is the ordinate of the lower right corner

d) Right is the abscissa of the lower right corner

3) AT is the area type. The most common AT collection is summarized as \{Static, Navigation, Query, List, Content, Operation, Form, and Page $\}$, where:

a) Static: represent the contents of this area are all static

b) Navigation: represent the contents of this area are all navigation links

c) Query: represent the contents of this area are conditions for query goal

d) List: represent the contents of this area are organized in a table

e) Content: represent the contents of this area are specific text

f) Operation: represent the contents of this area are operational button or link

g) Form: represent the contents of this area are a form

h) Page: represent the contents of this area correspond to another web page

4) $\mathrm{AC}$ is the Area Contents which describes the contents of area. Different AT corresponds to different AC description methods. Here gives the definitions of different AC.

Definition 6: SAC: Static Area Content is an $\Sigma$ (CD), where:

1) $\mathrm{CD}$ is the content description which explicit the static content this area should organize.

Definition 7: NAC: Navigation Area Content is an $\Sigma$ (ND, NR), where:

1) ND is the navigation description which explicit what kind of navigation links should appear in this area

2) NR is the relationship of the navigation.

Definition8: QAC: Query Area Content is an $\Sigma$ (FD, FR, QT, CL), where:

3) FD is a set of query field description. 
4) FR is the fields relationships which illustrate the constraint of different fields, include $\{$ And, OR $\}$

5) QT is query type, include \{Precise query, Fuzzy query\}.

6) $\mathrm{CL}$ is the corresponding list area, which indicates the results display area.

Definition9: FD: Field Description is an $\Sigma$ (FN, CT, Empty, Value, Default, FL), where:

1) $\mathrm{FN}$ is the name of the field.

2) $\mathrm{CT}$ is the field control type, the main control types in web application include \{Text, Checkbox, Radio, Textarea, Select\}

3) Empty indicates whether the field is required. Use 0 represents empty is permitted and 1 presents empty is not permitted.

4) Value defines the field content, for example the Select control must include some list options.

5) Default indicates the default value of the field.

6) FL is the field limit which explains the constraints of this field; include length period, character content, relationship with other field, etc.

Definition 10: LAC: List Area Content is an $\Sigma$ (Col, CSeq, Row, RSeq, Sort, PN, Refresh, RF), where:

1) Col defines the columns which should be shown in the list area.

2) CSeq defines the sequence of the columns in the list.

3) Row defines the default content of the list.

4) RSeq defines the sequence of the rows in the list.

5) Sort defines which columns should provide sort function.

6) $\mathrm{PN}$ is page number which defines the row number per page of the list.

7) Refresh defines whether the content of the list need refresh automatically or not.

8) RF is refresh frequency. If the list content need refresh automatically, this item defines the time interval of the refresh.

Definition 11: CAC: Content Area Content is an $\Sigma(\mathrm{CD})$, where:

1) $\mathrm{CD}$ is the content description which explicit the content this area should contain.

Definition 12: OAC: Operation Area Content is an $\Sigma$ (OS), where:

1) OS is a set of operation, and each operation is described as definition 13 .

Definition 13: BF: Button Function is an $\Sigma(\mathrm{ON}, \mathrm{PF}, \mathrm{OL})$, where:
1) $\mathrm{ON}$ is operation name.

2) $\mathrm{PF}$ is the ID of corresponding function page.

3) OL is operation logic which illustrates the operation business rules. If the button function will open a new page, the value is 0 .

Definition 14: FAC: Form Area Content is a set of FD, where:

1) FD is defined in definition 9.

Definition 15: PAC: Page Area Content is an $\Sigma(\mathrm{CD})$, where:

1) $\mathrm{CD}$ is the content description of the corresponding web page.

\section{MODEL EXAMPLE}

In this section, WASFM will be used in a real web system modeling. This system is a university information management system called DMIMS which is used by the teachers and staffs in the school to manage their daily work. This system's structure is shown in Figure 2 which has five top modules as 'System Management', 'Course Management', 'Reservation Management', 'Content Management' and 'Achievements Management'.

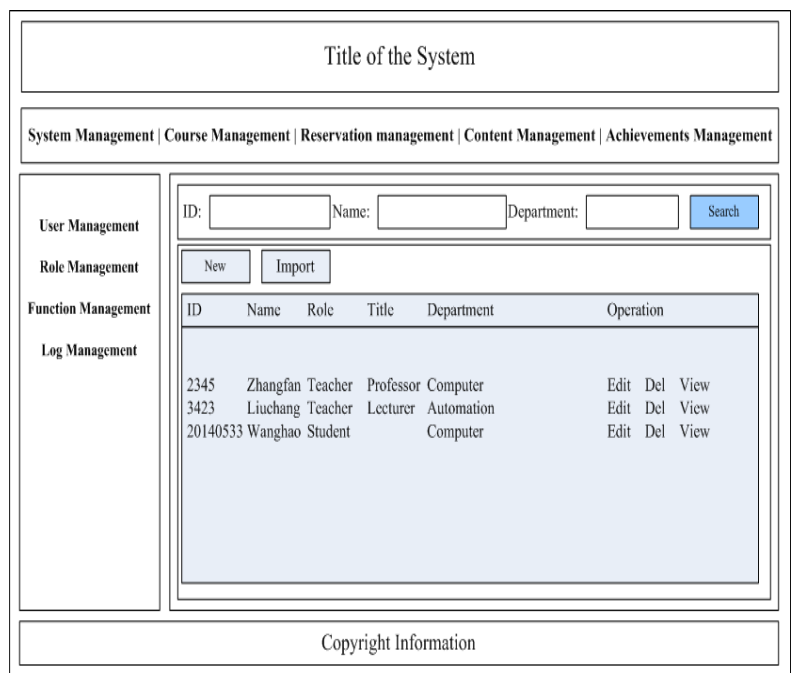

FIGURE II. FUNCTIONAL STRUCTURE OF A WAS EXAMPLE.

Each module has secondary functions such as 'System Management' module has 'User Management', 'Role Management', 'Function Management' and 'Log Management' four functions. Each secondary function corresponds to a web page which contains several functional areas. For example, 'User Management' functional page has four areas as 'Search', 'New', 'List', 'Operations', etc. Each button function will cause some process of system as 'add a new user', 'edit some course information', 'cancel some reservation', etc. When add a new user, there are some fields to be filled in, so the requirements should clear the fields contents, limitations and default.

Obviously, natural language is very difficult to describe the system functions accurately and completely, it is 
necessary to find some other formal methods to describe the system functional model. Based on the formal WAS functional model WASFM discussed above, we try to define the DMIMS functional model and give formal definitions of some important functions as below:

DMIMS $=\Sigma($ FR,NFR $)$

$\mathrm{FR}=\Sigma(\mathrm{CF}, \mathrm{PF}, \mathrm{BF})$

$\mathrm{CF}=\{\mathrm{CF} 1, \mathrm{CF} 2, \mathrm{CF} 3, \mathrm{CF} 4, \mathrm{CF} 5\}$

$/ /$ definition of catalog functions

$\mathrm{CF} 1=\Sigma($ System Management, 1,0$)$

$\mathrm{CF} 2=\Sigma($ Course Management, 1,0$)$

$\mathrm{CF} 3=\Sigma($ Reservation Management, 1,0$)$

$\mathrm{CF} 4=\Sigma$ (Content Management, 1,0$)$

$\mathrm{CF} 5=\Sigma($ Achievements Management, 1,0$)$

$\mathrm{PF}=\{\mathrm{PF} 1, \mathrm{PF} 2, \mathrm{PF} 3, \ldots \ldots\}$

$/ /$ definition of page functions

PF1 $=\Sigma$ (Main, http://10.104.16.2:8080/DMIMS, 0, PC1)

$/ /$ definition of the main page

$\mathrm{PC} 1=\{\mathrm{AD} 1, \mathrm{AD} 2, \mathrm{AD} 3, \mathrm{AD} 4, \mathrm{AD} 5\}$

$/ /$ definition of the main page area organization

$\mathrm{AD} 1=\Sigma(\mathrm{AD} 1, \quad(10,10,200,1024)$, Static, 'lay a static picture including the system's name')

//definition of title area

$\mathrm{AD} 2=\Sigma(\mathrm{AD}, \quad(210,10,280,1024)$, Navigation, ('level=1', null))

//definition of top navigation area

$\mathrm{AD} 3=\Sigma(\mathrm{AD} 3, \quad(290,10,800,300), \quad$ Navigation, ('level=2','list the next level

Catalog functions of the each top functions))

//definition of second navigation area

$\mathrm{AD} 4=\Sigma(\mathrm{AD} 4,(290,10,800,300)$, Page, 'function page of the AD3')

//definition of functional page area

$\mathrm{AD} 5=\Sigma(\mathrm{AD} 5,(810,10,840,1024)$, Static, 'Copyright information')

//definition of copyright area

$\mathrm{PF} 2=\Sigma \quad$ (User $\quad$ Management,/system/user/index.jsp, System management,

PC2)

//definition of the user management page

$\mathrm{PC} 2=\{\mathrm{AD} 6, \mathrm{AD} 7, \mathrm{AD} 8, \mathrm{AD} 9\}$

//definition of the user management page area organization
$\mathrm{AD6}=\Sigma(\mathrm{AD6},(10,10,200,680)$, Query,AC1 $)$

// definition of query area

$\mathrm{AD} 7=\Sigma(\mathrm{AD} 7,(210,10,300,680)$, Operation, $\mathrm{AC} 2)$

$\mathrm{AD} 8=\Sigma(\mathrm{AD} 8,(310,10,790,450)$, List,AC3 $)$

$\mathrm{AD} 9=\Sigma(\mathrm{AD} 9,(460,10,790,680)$, Operation, AC4 $)$

$\mathrm{BF}=\{\mathrm{BF} 1, \mathrm{BF} 2, \mathrm{BF} 3, \mathrm{BF} 4, \mathrm{BF} 5, \mathrm{BF} 6, \ldots \ldots\}$

//definition of button functions

$\mathrm{BF} 1=\Sigma($ Add User,PF3,0)

$\mathrm{PF} 3=\Sigma \quad$ (Add User,/system/user/addUser.jsp, User Management,PC3)

//definition of the add user page

$\mathrm{PC} 3=\{\mathrm{AD} 10, \mathrm{AD} 11\}$

$\mathrm{AD} 10=\Sigma(\mathrm{AD} 10,(10,10,200,680)$, Form,AC5 $)$

//definition of Form area

$\mathrm{AD} 11=\Sigma(\mathrm{AD} 11,(210,10,300,680)$, Operation, AC6 $)$

//definition of button area

$\mathrm{AC} 5=\{\mathrm{FD} 1, \mathrm{FD} 2, \mathrm{FD} 3, \mathrm{FD} 4, \mathrm{FD} 5, \ldots \ldots\}$

FD1 $=\Sigma($ UserId,Text,1,Null,Null,'Digital, 20’)

FD2 $=\Sigma($ Name, Text, 1,Null,Null,'Digital, 20')

FD3 $=\Sigma \quad$ (Role,Select,1,'Admin, Teacher, Student', Teacher, Null)

FD4 $=\Sigma$ (Title,Select,1,'Lecture, Associate Professor, Professor', Null, Null)

$\mathrm{FD} 5=\Sigma$ (Department,Select,1,'select from database department table', Null,

Null)

$\mathrm{AC} 6=\{\mathrm{BF} 7, \mathrm{BF} 8, \mathrm{BF} 9\}$

$\mathrm{BF} 7=\Sigma(\mathrm{OK}, \mathrm{Null}$, 'Add the new user information into database')

$\mathrm{BF} 8=\Sigma$ (Reset, Null, 'Reply to field values as the initial state')

$\mathrm{BF} 9=\Sigma$ (Cancel, Null, 'Cancel this operation')

The definitions above just give a small part description of the system, but it is obviously that the functions have been illustrated very clearly. According to this method, we can easily complete the definition of each function of the system.

With the definition of the web application system, first we can get a general understanding of the whole system such as how many main modules the system contains and the detail functions applied by each second module; secondly when to design the database and to develop the modules, we can refer to it to get the detail of each function. It will help the developer to do the work more standardized and reduce 
subsequent modifications; at last, for testing work, it gives constrains so will help the testers to design the cases fully.

\section{CONCLUSION}

This paper puts forward a new formal WAS functional model WASFM which can describe the web system functions completely and precisely. Formal definitions of the WAS functions have important significance, it can help designer to understand the system requirements accurately, help programmer clear the development task, help the tester grasp the details of the system and make it possible of programming and testing automatically.

\section{REFERENCES}

[1] Dzidek, W. J, Arisholm, E. and Lionel C, A Realistic Empirical Evaluation of the Costs and Benefits of UML in Software Maintenance, IEEE Transactions on Software Engineering, Vol. 34(3), pp. 407-432, 2008

[2] TANG Shengqun, LIU Wei and MA Jie, Inferring Requirement Goals from Model Implementing by UML, Computer Engineering, Vol. 28(3), pp. 77-79, 2002.

[3] Feldmann, Clarence G. The practical guide to business process reengineering using IDEF0. Dorset House Publishing, NY, 1998.

[4] Rozilawati Razali, Parnian Najafi, Seyed Hadi Mirisaee: Combining Use Case Diagram and Integrated Definition's IDEF0 - A Preliminary Study. International Conference on Software Engineering and Data Mining, 2010.

[5] Atoosa Jalashgar. Function-Oriented System Analysis: Putting the GTST, MFM and HMG Methods into Perspective. Proc. of the 5th Int'L Workshop on Functional Modeling of Complex Technical System, Paris-Troyes, France, 1997, p.51-7. 